\title{
NEUROSCIENCE
}

\section{Sex-specific effects of Tac2 blockade on fear memory formation}

Florido, A. et al. Nat. Commun. 12, 2496 (2021)

The capability to form a fear memory enables us to learn from past traumatic experiences and modify our decisions to avoid similar situations in the future. When excessive, or triggered inappropriately, fear response contributes to the development of anxiety disorders such as panic disorder, phobia, and posttraumatic stress disorder (PTSD); a better understanding the mechanisms mediating fear consolidation could guide the development of targeted approaches for the treatment of fear-related disorders.

In previous work, Raül Andero and colleagues at Emory University School of Medicine demonstrated that neurokinin $\mathrm{B}(\mathrm{NkB})$, encoded by $T a c 2$, and its specific receptor, $\mathrm{Nk} 3 \mathrm{R}$, are involved in the consolidation of fear memories. They also showed that the pharmacological blockade of the Tachykinin 2 (Tac2) circuit, located in the central amygdala (CeA), decreases fear memory consolidation in male mice, making osanetant, the Nk3R antagonist used to block the pathway, a promising agent to block fear memory consolidation after exposure to a trauma and prevent PTSD.

However, in a new study published in Nature Communications, Andero, who works now at the Institute of Neuroscience, Autonomous University of Barcelona, and his team show that osanetant's effects on fear memory consolidation depend on sex. By revealing that the same drug can have opposite effects on memory between male and female mice, the study further supports the need to include both sexes in neuroscience studies to produce more representative and translational findings.

"The study of sex differences is still not fully considered in the scientific community researching the brain-including memoryalthough women are more likely to present a fear-related disorder. In the last years, researchers have published 5.5 studies in males per 1 in females pointing out the evident and growing need to change our approach to neuroscience by including

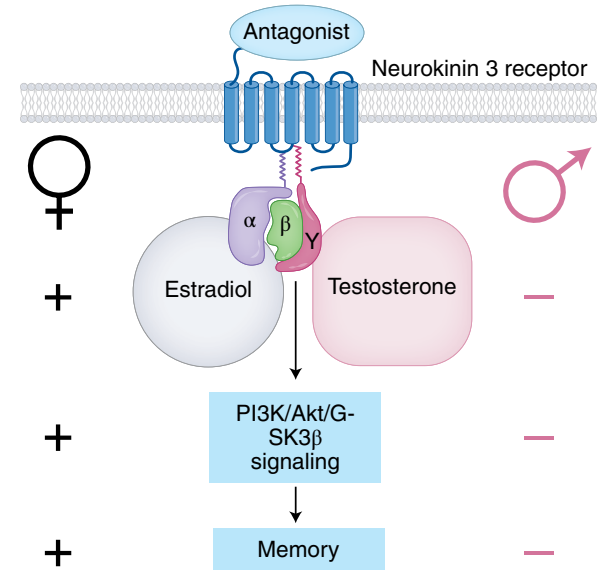

Inactivation of Tac2 pathway impairs and increases fear memory consolidation in males and females, respectively. Adapted from Florido et al (2021), Springer Nature. Credit: Marina Spence

female subjects at all levels of research," write the investigators in their report.

The team submitted female and male C57BL/6J mice to a cued-fear conditional paradigm in which animals were trained to associate a cue (tone) with a fear-inducing stimulation (foot shock), before injection of osanetant $(5 \mathrm{mg} / \mathrm{Kg}$ ) or vehicle $30 \mathrm{~min}$ after fear acquisition. A fear expression test performed $24 \mathrm{~h}$ after fear acquisition revealed that osanetant-treated female mice presented higher rates of freezing than the vehicle-treated female mice, whereas osanetant-treated males showed decreased consolidation.

Hypothesizing that sex hormones could contribute to these sex-specific effects, the investigators divided the female mice according to their estrous cycle stage on the day of fear acquisition and drug administration, and repeated the experiment. Analysis of freezing behavior showed that osanetant increased fear memory consolidation in females when the manipulations were made during proestrus-a stage presenting high concentrations of estradiol-but had no effect during estrus, metestrus or diestrus. Next, Florido et al. measured the effects of osanetant on sex hormones levels. They showed that osanetant reduced testosterone levels in male mice during the consolidation window of memory and increased estradiol concentrations in female mice in proestrus, whereas it had no effect in females in metestrus. In summary, osanetant produced a sex opposite regulation in males and proestrous females, which might explain how the drug influences the consolidation of fear memory in males and females in totally contradictory ways.

Further experiments revealed sex-based differences in how osanetant regulated the Akt/GSK3 $\beta / \beta$-Catenin pathway in the amygdala of male mice and proestrus female mice during memory consolidation, with an increase of GPCR-related genes in females in proestrus but a decrease of GPCR-related genes in males; this result is also in line with the behavioral outcomes.

"These data thereby provide evidence that there exist opposite sex effects after a drug administration in behavioral and molecular mechanisms in memory formation through the involvement of the Akt/GSK3- $\beta / \beta$-Catenin signaling pathway in Nk3R-positive neurons of the CeA in interaction with sex hormones," conclude the investigators, before adding that their discovery proposes a new perspective on why and how memory processes differ between females and males. These findings could also guide the development of more personalized treatments for anxiety disorders and PTSD, taking sex as an important biological variable.

Alexandra Le Bras

Published online: 17 May 2021

https://doi.org/10.1038/s41684-021-00778-z 